\title{
Laparoscopic management of endometrial stromal sarcoma in young: a case report
}

\author{
Shailesh Puntambekar ${ }^{1}$, Shetty Theertha Shankar ${ }^{2 *}$, Arjun Goel ${ }^{1}$, Shakti Panchal ${ }^{2}$
}

\author{
${ }^{1}$ Department of Surgery, Galaxy Care Multispeciality Hospital Pvt. Ltd., Pune, Maharashtra, India \\ ${ }^{2}$ Department of Gynaecology, Galaxy Care Multispeciality Hospital Pvt. Ltd., Pune, Maharashtra, India
}

Received: 17 February 2020

Revised: 20 March 2020

Accepted: 27 March 2020

\section{*Correspondence:}

Dr. Shetty Theertha Shankar,

E-mail: shettytheerthas@gmail.com

Copyright: (C) the author(s), publisher and licensee Medip Academy. This is an open-access article distributed under the terms of the Creative Commons Attribution Non-Commercial License, which permits unrestricted non-commercial use, distribution, and reproduction in any medium, provided the original work is properly cited.

\begin{abstract}
Endometrial stromal sarcoma (ESS) is a case malignancy and accounts for $0.2 \%$ of all uterine malignancies and $10 \%$ of all uterine sarcomas. In the present case, an unmarried 27-year-old woman presented with complaints of on and off episodes of pain in the lower abdomen. On examination, a diffuse oval mass was felt occupying the hypogastrium, iliac and lumbar regions and extending up to the umbilicus. CT scan impression was given as? Complex retroperitoneal mass? solid cystic ovarian mass with grade I right hydro nephrosis. On laparoscopy, a huge mass measuring approximately $10 \times 10 \mathrm{~cm}$ was noted arising from the uterus. Laparoscopic myomectomy was done and the specimen was retrieved in a bag and the tissue was sent for histopathological examination. The postoperative clinical course was uneventful. The patient was advised to undergo oocyte cryopreservation following which she was advised hysterectomy with bilateral salphingo-oophorectomy. Pelvic radiation was advised thereafter. ESS is a rare malignant tumor, which on histopathological examination reveals sheets of cells and endometrial stromal cell differentiation. Hysterectomy with bilateral salpingo-oophorectomy is optimum initial therapy. Radiotherapy is chosen when the tumour is inadequately excised or the pelvic disease is locally recurrent. Low grade ESS is a rare form of uterine sarcoma usually seen between 40-60 years of age with limited available data regarding the clinical course and management. Early diagnosis and management is associated with a favourable prognosis and outcome.
\end{abstract}

Keywords: Malignancy, Sarcoma, Young

\section{INTRODUCTION}

Endometrial stromal sarcoma (ESS) is a rare malignancy and accounts for $0.2 \%$ of all uterine malignancies and $10 \%$ of all uterine sarcomas. ${ }^{1}$

Histological classification of uterine sarcomas includes carcinosarcomas (malignant mesodermal mixed tumors), leiomyosarcomas, endometrial stromal sarcomas and undifferentiated sarcomas. ${ }^{2}$

As per 2014 World Health Organization (WHO) classification, endometrial stromal sarcoma can be classified into four types: endometrial stromal nodule (ESN), low-grade endometrial stromal sarcoma (LGESS), high-grade endometrial stromal sarcoma (HGESS), and undifferentiated uterine sarcoma (UUS). ${ }^{3}$

Soft tissue sarcomas occur at any anatomical site and have a varied range of behaviours, hugely dependent on the histologic subtype and grade of tumour. ${ }^{4}$

Aetiology of these tumours is poorly understood, although there may be some association with raised or unopposed oestrogen levels, treatment with tamoxifen, obesity and diabetes. 
Low-grade endometrial stromal sarcomas occur in women between 40 and 55 years of age and more than $50 \%$ of patients are premenopausal. ${ }^{2}$

Patients commonly present with abnormal uterine bleeding, pelvic pain, and dysmenorrhea, but about $25 \%$ are asymptomatic.

Generally, it is not conducive to distinguish a sarcoma from a leiomyoma by imaging and clinical characteristics and uterine sarcomas are generally diagnosed after a surgical procedure performed for a mass presumed to be benign. ${ }^{5}$

Recommendations for treatment include hysterectomy with bilateral salphingo oophorectomy as the primary treatment modality of ESS. ${ }^{6}$

Some researchers have recommended preservation of ovarian function, particularly in younger women.

\section{CASE REPORT}

An unmarried 27-year-old woman presented to the outpatient department with complaints of on and off episodes of pain in the lower abdomen since 4 months which had aggravated since 3 days.

She complained of a mass in the lower abdomen which had undergone a sudden increase in size.

She gave a history of regular menstrual cycles with no history of menorrhagia or dysmenorrhoea.

On examination, a diffuse oval mass was felt occupying the hypogastrium, iliac and lumbar regions and extending up to the umbilicus.

Margins were well made out.

Mobility was present transversely.

Mild tenderness and guarding were present.

CA-125 levels were $106 \mathrm{U} / \mathrm{ml}$. All other laboratory tests including hormonal tests were unremarkable.

CT scan revealed multiloculated mixed density abdominopelvic mass measuring $23 \times 22 \times 11 \mathrm{~cm}$ with solid and cystic fluid filled areas causing a mass effect on the right and left colon, right ureter and IVC (Figure 1: i, ii, iii, iv).

Sigmoid colon was sandwiched between uterine fundus and instead of abuts, mass was abutting the right ovary and anterior uterine surface. There was no evidence of ascites or enlarged nodes.

There was grade I right hydronephrosis.
Impression was given as? Complex retroperitoneal mass,? solid cystic ovarian mass with grade I right hydronephrosis.
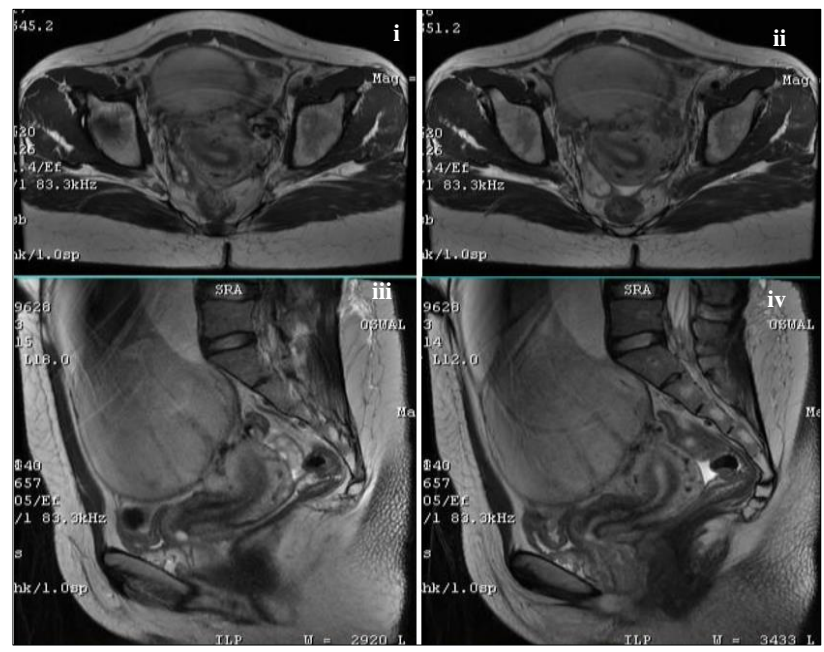

Figure 1: (i, ii, iii, iv) Computed tomography scan suggestive of a complex abdominopelvic mass measuring $23 \times 22 \times 11 \mathrm{~cm}$ with solid and cystic fluid filled areas causing a mass effect on the right and left colon, right ureter and IVC.

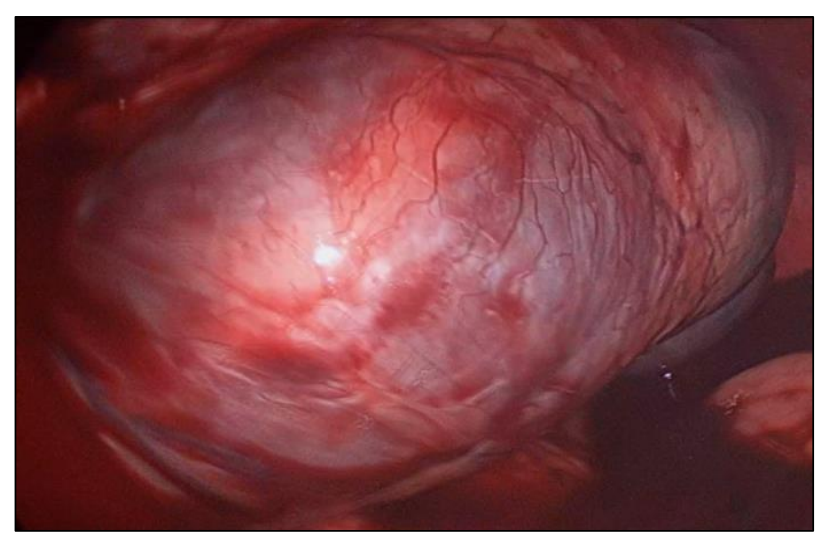

Figure 2: $10 \times 10 \mathrm{~cm}$ mass arising from the uterus seen intraoperatively.

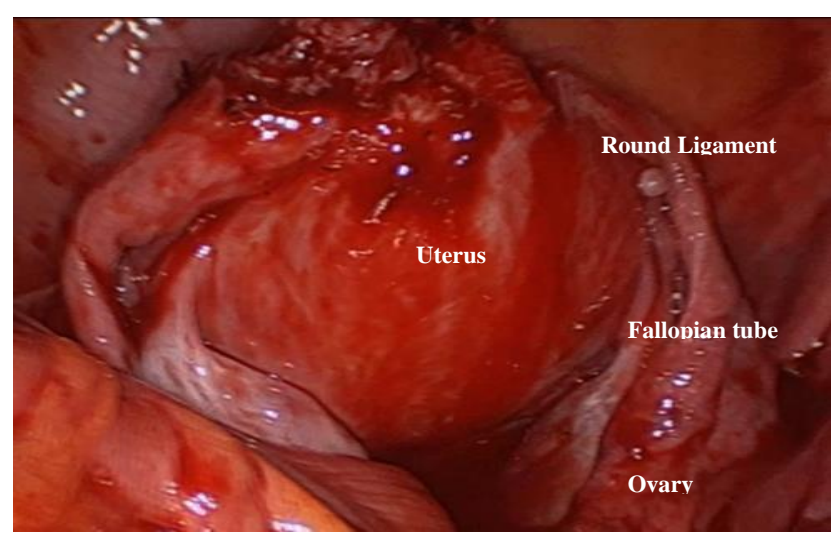

Figure 3: Intra-operative following myomectomy. 


\section{Intraop findings}

On laparoscopy, a huge mass measuring approximately $10 \times 10 \mathrm{~cm}$ was noted arising from the uterus (Figure 2) Considering the woman was unmarried, a laparoscopic myomectomy was done (Figure 3) and the specimen was retrieved in a bag through a small transverse incision and the tissue was sent for histopathological examination.

\section{Histopathology}

The histopathological report confirmed the diagnosis of low grade endometrial stromal sarcoma (LGESS). (Figure 4).

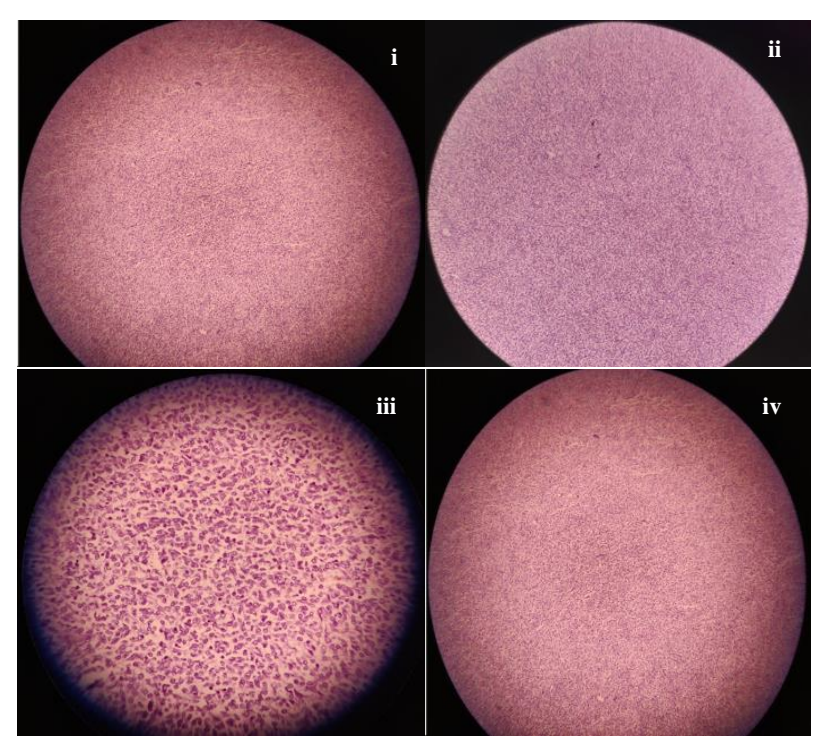

Figure 4: (i, ii, iii, iv) Histopathological endometrial stromal sarcoma.

\section{Immunohistochemistry}

Immunohistochemical study of the tissue showed the tumor cells were focally positive for CD10 (strong), cyclin D (weak in 10\%), Er (weak in approximately $20 \%$ ), MIB-1 (approximately $6 \%$ on an average) and were negative for smooth muscle actin (SMA), $\mathrm{H}$ caldesmon, WT-1 and desmin, leading to the probable diagnosis of ESS. The post-operative clinical course was uneventful. The patient was advised to undergo oocyte cryopreservation following which she was advised hysterectomy with bilateral salphingo-oophorectomy. Pelvic radiation was advised thereafter.

\section{DISCUSSION}

ESS is a rare malignant tumor, which on histopathological examination reveals sheets of cells and endometrial stromal cell differentiation.

LGESS presents with infiltrating margins coupled with extensive worm-like lymphatic and venous vessel invasion. $^{1}$
LGESS usually presents in patients between ages 42 and 53 , in our case-report, the patient was a 27 -year-old woman.

Ashraf-Ganjoei et al, also observed vaginal bleeding as the presenting complaint in $86 \%$ of the patients. ${ }^{7}$

The differential diagnoses are leiomyoma, uterine leiomyosarcoma or other sarcomas or retroperitoneal masses.

Immunohistochemical features of ESS are similar with leiomyoma with expression of SMA, and desmin. CD10 immunoreactivity is a positive predictive marker for ESS which is sensitive and useful for the diagnosis of normal endometrial stroma and endometrial stromal neoplasms.

Hysterectomy with bilateral salpingo-oophorectomy is optimum initial therapy. Radiotherapy is chosen when the tumour is inadequately excised or the pelvic disease is locally recurrent.

Owing to the rarity of endometrial stromal sarcomas, evidence on their clinical behaviour and treatment strategies is limited. ${ }^{6}$

Case reports and retrospective case series by academic institutions with a small number of patients accrued over long periods account for the data available. In addition, there are no large-scale studies on endometrial stromal sarcomas alone. Low-grade ESS has an inert course associated with high overall survival which can be amenable to resection and/or endocrine therapy. ${ }^{4}$

Role of adjuvant RT and retrospective series on local therapy has not been demonstrated separately for HGESS, HGUS and low-grade ESS. Low-grade sarcoma patients may be followed up every 4-6 months for the first 3-5 years to rule out local recurrences and then yearly.

High-grade tumors may be followed-up every 3-4 months for the first 2-3 years, twice a year for the next 2-3 years, and then yearly.

\section{CONCLUSION}

This case report highlights the presence of low grade ESS in a young patient who presented with a mass in the lower abdomen as the only presenting complaint. Low grade ESS is a rare form of uterine sarcoma usually seen between 40-60 years of age with limited available data regarding the clinical course and management.

Early diagnosis and management are associated with a favourable prognosis and outcome.

Funding: No funding sources

Conflict of interest: None declared

Ethical approval: Not required 


\section{REFERENCES}

1. Jassal CD, Patnaik BL, Divya A, Prasad S. Lowgrade endometrial stromal sarcoma in young age: A clinicopathological report. The J Obstet Gynecol India. 2012;62(1):73-5.

2. Prat J, Mbatani N. Uterine sarcomas. Inter J Gynecol Obstet. 2015;131:S105-10.

3. Eamudomkarn N, Itarat $\mathrm{Y}$, Kleebkaow $\mathrm{P}$, Kietpeerakool C. A case report of high-grade endometrial stromal sarcoma: a rare cause of abnormal uterine bleeding in a young woman. Case Reports Obstet Gynecol. 2018;2018.

4. Benson $\mathrm{C}$, Miah AB. Uterine sarcoma-current perspectives. Inter J Women's Health. 2017;9:597.

5. Brohl AS, Li L, Andikyan V, Običan SG, Cioffi A, Hao K, et al. Age-stratified risk of unexpected uterine sarcoma following surgery for presumed benign leiomyoma. The Oncol. 2015;20(4):433.

6. Chan JK, Kawar NM, Shin JY, Osann K, Chen LM, Powell CB, et al. Endometrial stromal sarcoma: a population-based analysis. $\mathrm{Br} \quad \mathrm{J}$ Cancer. 2008;99(8):1210-5.

7. Ashraf-Ganjoei T, Behtash N, Shariat M, Mosavi A. Low grade endometrial stromal sarcoma of uterine corpus, a clinico-pathological and survey study in 14 cases. World J Surg Oncol. 2006;4(1):50.

Cite this article as: Puntambekar S, Shankar ST, Goel A, Panchal S. Laparoscopic management of endometrial stromal sarcoma in young: a case report. Int J Reprod Contracept Obstet Gynecol 2020;9:2144-7. 